\title{
Synthèse des connaissances et quelques acquis de recherche sur le sésame (Sesamum Indicum L.) au Sénégal
}

\author{
Birama SENE ${ }^{1,2^{*}}$ Fallou SARR ${ }^{1}$, Diégane DIOUF ${ }^{2}$, Mamadou Salif SOW ${ }^{1}$, \\ Djibril TRAORE ${ }^{1}$, Amadou KANE $^{1}$ et Marème NIANG ${ }^{3}$ \\ ${ }^{l}$ Institut de Technologie Alimentaire (ITA), Route des Pères Maristes, Hann, Dakar, BP 2765 Dakar, Sénégal. \\ ${ }^{2}$ Université Cheikh Anta DIOP de Dakar, Avenue Cheikh Anta Diop, BP : 5005 Dakar, Fann, Sénégal. \\ ${ }^{3}$ Centre d'Etudes Régional pour l'Amélioration de l'Adaptation à la Sécheresse (CERAAS), BP 3320, \\ Thiès-Escale, Thiès, Sénégal. \\ "Auteur correspondant ; E-mail: senebiramam2chimie@gmail.com
}

\section{RESUME}

Plante herbacée annuelle, à plusieurs propriétés, le sésame (Sesamum Indicum L.) a été introduit au Sénégal pour une rotation et une diversification des cultures afin d'augmenter la productivité et les revenus des populations rurales et contribuer à la sécurité alimentaire. Afin d'améliorer les conditions de culture du sésame, des recherches ont été entreprises sur cette plante oléagineuse. L'objectif de cette étude était de faire une revue de la composition, l'état de la recherche et les utilisations du sésame au Sénégal. Les recherches bibliographiques ont permis de montrer quelques acquis au plan de la recherche. C'est le cas de la connaissance des conditions pédoclimatiques et les techniques de culture de cette plantation au Sénégal. Les paramètres agro-morphologiques de la plante et l'amélioration variétale par les phénomènes de mutation ou de mycorhization ont également été étudiés. Les futurs programmes de recherche devraient mettre l'accent sur la composition biochimique, nutritionnelle et minérale, ainsi que sur son utilisation et sa biotransformation, de même que sur les aspects de défense des cultures et phytosanitaires de ces produits dérivés.

(C) 2018 International Formulae Group. All rights reserved.

Mots clés: Sésame, synthèse, acquis de recherche, composition, Sénégal

\section{Knowledge synthesis and acquired research of sesame (Sesamum Indicum L.) in Senegal}

\begin{abstract}
Sesame (Sesamum Indicum L.) is an annual herbaceous plant that has been introduced in Senegal for the sake of crop rotation and diversification in order to increase productivity as well as income of rural population but also for food safety. In order to improve the growing conditions of sesame, research were undertaken on this oleaginous plant. The literature revealed that some knowledge were available on the pedoclimatic and on the growing conditions of the crop. Agro-morphological parameters of the crop and variety improvement by means of mutation breeding or by mycorhization technique have been studied. In
\end{abstract}


Senegal, future research programs on sesame should stress the biochemical, mineral composition, nutritional and the biotransformation aspects of these derived products and explore the crop protection domain.

The objective of this study is to review the composition, the state of research and the uses of sesame in Senegal.

(C) 2018 International Formulae Group. All rights reserved.

Keywords: Sesame, synthesis, acquired research, composition, Senegal.

\section{INTRODUCTION}

Le sesame (Sesamum Indicum L.) est une ancienne et importante culture oléagineuse, il est cultivé principalement dans les régions tropicales et subtropicales d'Asie, d'Afrique et d'Amérique du Sud. Il appartient à la classe des Dicotylédones vrais ou Eudicotylédones, à l'ordre des Lamiales, à la Famille des Pédaliacées et au genre des sésamées (Weiss, 2000 ; Stevens, 2011). Il présente une large diversité de génotypes (Weiss, 2000 ; Söğüt, 2008).

Le sésame est une plante herbacée annuelle, à port érigé, d'une hauteur de 0,5 à 2,5 $\mathrm{m}$ dans les conditions optimales de croissance. La tige, de section carrée longitudinalement cannelée, est de couleur verte, rarement pourpre. Son diamètre basa1 varie de 1 à 3 . Elle peut être glabre, veloutée ou poilue. Ce trait poilu de la tige et des rameaux permet un groupage de variétés (Kafiriti et Deckers, 2001). Les feuilles inférieures sont opposées, larges $(12 \times 8 \mathrm{~cm})$, grossièrement lobées et à long pétiole $(5 \mathrm{~cm})$. Par contre, les feuilles supérieures sont alternes ou sub-opposées, ou étroites $(9 \times 2$ $\mathrm{cm}$ ), avec une phyllotaxie particulière (Weiss, 2000 ; Kafiriti et Deckers, 2001). Les feuilles sont de couleur verte terne avec des poils et des stomates sur les deux faces (Weiss, 2000).

Son système racinaire est de type mixte avec un pivot à croissance initiale rapide pouvant atteindre $90 \mathrm{~cm}$ et un réseau dense de racines superficielles (lacis de radicelles) peu développées (Diouf, 2002). Selon Weiss (2002), l'élongation racinaire contribue aux stratégies d'évitement de la sécheresse et la croissance initiale rapide du pivot serait due au prélèvement d'une quantité de phosphore par les racines secondaires à 5 et 8 centimètres du sol.
En Afrique, on note 17 espèces de Sesamum dont deux qui poussent aussi à l'état sauvage en Inde. L'espèce $S$. indicum, est également connue sous le synonyme Sesamum orientale L. On peut citer aussi d'autres synonymes moins connus de $S$ indicum comme S. edule, S. luteum, S. oleiferum, $S$. africanum, S. foetidum (Demol et al., 2002). Le genre Sesamum comporte de très nombreuses variétés qui diffèrent par leurs dimensions, leur forme, le type de croissance, la couleur des fleurs, le calibre, la couleur et la composition des graines (Demol et al., 2002).

Au Sénégal, le sésame a été réintroduit en 1985 en moyenne Casamance à partir de la Gambie par le biais de l'Association Africaine de la Jeunesse Agricole et Culturelle/Comité de Lutte pour la Faim (AAJAC/COLUFIFA) (CRS, 1999). Au Sénégal, le sésame est devenu une alternative pour plusieurs zones de culture, non seulement par ses faciles conditions de culture, sa résistance à la sècheresse et sa demande mondiale qui ne cesse d'augmenter, mais surtout pour la volonté de diversification de la seule culture de rente (arachide). Ceci du fait des aléas climatiques qui ont occasionné une dégradation de l'environnement agraire et la baisse du niveau de production des cultures vivrières (mil, sorgho, maïs), et de la principale culture de rentre (arachide) au Sénégal.

Ainsi, la question de sécurité alimentaire reste un défi urgent à relever ; objectif prioritaire que se sont fixés les pouvoirs publics et les institutions de recherche avec l'adoption d'une politique de mise au point de technologies agricoles appropriées, de production de semences adaptées aux diverses zones agro-écologiques et à terme l'optimisation et la stabilisation des 
rendements agricoles. Dans cette optique, l'amélioration du sésame revêt un intérêt majeur du fait de sa grande importance socioéconomique au Sénégal à l'image de l'ensemble de la zone sahélienne. Des acquis importants ont été obtenus. Cette synthèse bibliographique présente l'état de la recherche sur le sésame au Sénégal, sa production au Sénégal et dans le monde, son utilisation mais surtout sa composition chimique et biochimique. Il s'agit d'une étude générale destinée à mieux appréhender le potentiel et les atouts de cette spéculation.

\section{ETAT DE LA RECHERCHE SUR LE SESAME AU SENEGAL}

Pendant que la demande en graines de sésame sur le marché international est de plus en plus forte, cette plante oléagineuse reste toujours méconnue dans beaucoup de zones au Sénégal. Les recherches concernant sa composition chimique, ses qualités nutritionnelles et son utilisation sont toujours considérées comme à leur début. A notre connaissance, les quelques recherches effectuées sur le sésame au Sénégal ont toutes été axées soit sur les conditions agromorphologiques et pédoclimatiques de la plante, soit sur l'amélioration variétale par les phénomènes de mutation ou de mycorhization après celles effectuées par le CERAAS et qui ont porté sur l'effet de la pression osmotique sur la germination des semences de sept variétés de sésame nouvellement introduites. Ces recherches visaient plus l'augmentation des rendements que la connaissance de la composition chimique et biochimique des graines puisque le sésame était destiné à la commercialisation. C'est ainsi qu'une synthèse bibliographique sur la culture du sésame (Diouf, 2004), une fiche technique (Diouf et al., 2004) et un guide (Ndiaye et al., 2005) comme support à la diffusion des connaissances sur la conduite de cette culture ont été élaborés. C'est recherches ont été soutenues par une caractérisation variétale où deux types de variétés sésame ont été identifiées : les variétés ramifiées (38-1-7, 3215, Jaalgon 128 et primoca) selon (Guèye,
2002) et la variété tolérante à la sécheresse (Ceraas-1-98) d'après Pelletier, (2003). En outres, d'autres études portant sur la technologique de l'administration de la dose optimale en intrants (Ndiaye, 2004), la date de semis et la densité par hectare (Bèye, 2003 ; Niang, 2004) ont été effectuées. De même que le comportement aux conditions de stress hydrique ou non, les besoins en eau et les performances agronomiques de quelques variété cultivées en milieu paysan (Ceraas-198, 32-15, 38-1-7, Jaalgon 128, Cross $n^{\circ} 3$, Yendev 55 et Primoca) ont été déterminés (Diouf, 2002; Diouf et al., 2002 ; Fofana, 2004 ; Boureima, 2005). Cette situation de la recherche sur le sésame peut être expliquée par le fait que le sésame n'est pas une culture pour laquelle les centres de recherches du système CGIAR (Consultative Group on International Agricultural Research) ont un mandat (Ashri, 2001). En outre, sa culture se fait le plus souvent dans les pays en développement avec des ressources disponibles limitées pour la recherche à long terme (Ashri, 2007); c'est le cas au Sénégal.

\section{PRODUCTION DU SESAME DANS LE MONDE ET AU SENEGAL}

La production de graines de sésame augmente à l'échelle mondiale. Le sésame se classe au neuvième rang parmi les treize premières cultures oléagineuses qui constituent $90 \%$ de la production d'huile comestible dans le monde (Saha et al., 2014). Cet état s'explique par la forte demande mondiale en graines de sésame qui, elle se justifie par la bonne teneur en huile $(50 \%)$ et en protéines $(25 \%)$ de la graine d'après Burden, (2005) et par la présence des antioxydants (sesamine, sésamoline et sésamol) qui rendent l'huile plus stable. La récolte est à la fois utilisée pour les semences et dans la cuisson après une extraction de l'huile des graines. Ce double usage des récoltes de sésame illustre les grandes superficies cultivées dans le monde. Ainsi, 2 à 2,5 million d'ha sont emblavés en Inde (Weiss, 2000 ; Valarmathi et al., 2003), un million d'ha en Chine, 0,6 millions d'hectares 
en Birmanie, 0,5 million d'hectares au Soudan et 0,25 million d'hectares au Mexique (Mémento de l'agronome, 2002). La productivité mondiale dépend des pays et des zones de cultures. L'Inde occupe la première place avec une productivité du sésame de 0,33 tonnes / ha par rapport à la moyenne mondiale de 0,44 tonnes / ha. Elle est suivie par la Chine (19,9\%), le Myanmar (17,3\%), le Soudan $(5,9 \%)$, l'Ouganda (4,9\%), l'Éthiopie $(4,7 \%)$ le Nigeria $(2,9 \%)$, le Bangladesh $(1,4 \%)$ et le Pakistan $(0,8 \%)$ (Raghav et Agarwa, 2013). L'Afrique n'est pas restée en rade par rapport à la dynamique d'augmentation de la production mondiale. Elle est passée au deuxième rang mondial en augmentant sa production de $25 \%$ avec ses sept pays appartenant au groupe des plus grands exportateurs de graines de sésame dans le monde et dont deux se trouvent en Afrique de l'Ouest, le Nigéria avec 39800 tonnes et le Burkina Faso avec 18107 tonnes (FAO, 2004).

Au Sénégal, la pratique de la culture est relativement ancienne mais elle avait connu un ralentissement avant d'être réintroduite en 1985 par l'Association africaine de la jeunesse agricole et culturelle/Comité de lutte pour la fin de la faim (AAJAC/COLUFIFA) en Moyenne Casamance (CRS, 1999). Depuis, une augmentation de l'engouement de la culture est notée au niveau des populations. Cette rué vers cette nouvelle plante s'est manifesté par l'étendue des superficies emblavées et les productions qui ne cessent de croître. Elles passent respectivement de 1615 ha en $1994 / 95$ à 52419,5 ha en 2005/2006. La production est passée de $640 \mathrm{~T}$ en 1994 à $31839 \mathrm{~T}$ en 2006 dans les régions du centre (Kaolack) et du Sud à Sud-Est (Kolda et Tambacounda) du pays (Daps, 2006). Le Tableau 2 montre les taux de production de sésame selon les régions. On voit que sur les 14 régions que compte le Sénégal, 5 assurent presque la totalité de la production du sésame avec un taux de $94 \%$. La production est plus importante dans les régions de Kaolack (42\%) et de Kolda (35\%). Ces données montrent qu'un travail sur la connaissance de la plante, ces vertus et ses utilités du point de vue alimentaire, cosmétique, pharmaceutique mais aussi médicinal mérite d'être opéré en vue d'une vulgarisation nationale. Il ressort de ces données que l'intégration du sésame dans le système de culture n'est pas effective dans certaines régions au Sénégal. Cette évolution de la production est la résultante de deux facteurs : l'intérêt que les populations rurales accordent à cette nouvelle spéculation tant pour la facilité de sa culture et le faible coût de ses intrants d'une part, et la volonté de l'Etat du Sénégal de développer la filière sésame d'autre part. Mais la culture de sésame s'est vite heurtée à des contraintes majeures liées notamment à la production car la réintroduction a été faite de manière informelle, donnant ainsi en milieu paysan des rendements encore faibles dépassant rarement les moyennes des 300 à 400 kg/ha (Boureima, 2012), avec une exception faite en 2010 ou le rendement était à $416 \mathrm{~kg} / \mathrm{ha}$ (Daps, 2011).

Les différentes variétés de sésame cultivées sont présentées dans le Tableau 3 avec quelques caractéristiques physiologiques et morphologiques mais aussi les rendements obtenus en station et au champ.

\section{COMPOSITION DE LA GRAINE}

$\mathrm{La}$ distribution des composants bioactifs dans les graines de sésame représente sa valeur alimentaire élevée. Le sésame (Sesamum indicum L.) est une plante oléagineuse alimentaire présentant une valeur nutritionnelle élevée (acides gras : 45 à 55\%, protéines : 19 à $25 \%$, minéraux : $\mathrm{Ca}, \mathrm{P}, \mathrm{Mg}$, $\mathrm{Fe}, \mathrm{Zn}$ ) (Nzikou et al., 2010; Rizki et al 2017 ; Deme et al., 2017 ; Sene et al., 2017). Les vitamines (B et E) et les antioxydants des graines de sésame ont été aussi étudiés par Borchani et al. (2010) et Yogranjan et al., (2014). D'autres recherches plus récentes ont été menées au Maroc et ont mis en évidence d'autres éléments minéraux sur des génotypes de sésame marocains comme le sélénium $(\mathrm{Se})$ et le potassium (K) (Rizki et al., 2017). Selon ces auteurs, le sélénium, considéré comme bénéfique pour les consommateurs est très présent dans la composition minérale des 
graines de sésame à l'image du calcium et du phosphore; ceci renforce l'importance pharmaceutique et nutritionnelle des graines de sésame. Cette composition dépend de la variété, de l'origine géographique, des pratiques culturales, de la date de récolte et du produit étudié (graines, huile tourteau de sésame). Du point de vue de la composition protéino-énergétique, les travaux de Gandhi et Srivastava (2007) et de Nzikou et al. (2009) ont donné respectivement $26.94 \%$ et $20.00 \%$ de protéines et $48.21 \%$ et $54.00 \%$ de matières grasses. Etant donné que les matières grasses fournissent plus d'énergie selon les coefficients d'Atwater le sésame pourrait alors être un remède dans l'éradication de la malnutrition protéino-énergétique. Cette forte composition en protéines et en matières grasses a été aussi montrée par les travaux d'Hassan (2012), d'Ogbonna et Ukaan (2013) et ceux de Gharby et al. (2015). En outre, Rivas et al (1981) ont rapporté dans la plupart des protéines dans la farine de sésame : 8,6\% d'albumine, $67,3 \%$ de globuline et $1,4 \%$ de prolamine et $6,9 \%$ de glutéline. De la même façon qu'on peut utiliser les graines de sésame pour éradiquer la malnutrition protéinoénergétique, la composition en acides aminés de la protéine de sésame a montré qu'il peut être utilisé comme une base de régime, complétant les céréales et les légumineuses. La farine issue des graines de sésame contient des protéines à des quantités élevées, du soufre total contentant les acides aminées méthionine et cystine alors que beaucoup des protéines végétales ont des teneurs faibles en acide aminé total soufré comme la protéine de la farine de maïs (Brito et Nuñez, 1982), soja (Iwe et al., 2001), le riz blanchi (Peter, 2007) et la farine de blé (Peter, 2007). A côté de ces acides aminés, une forte fraction d'antioxydants, composée de la sésamine et de la sésamoline avec tous ces produits dérivés a été trouvée (Abou-Garbia et al., 2000 ; Lee et Choe, 2012 ; Yogranjan et al., 2014 ; Rizki et al., 2015). C'est cette composition qui lui confère sa stabilité et sa résistance au rancissement. D'après Biswa et al. (2001), la teneur en cendres $(4,5 \%)$ lui confère un taux de matière minérale élevé. Les graines de sésame sont une excellente source de cuivre et de calcium. Elles sont également riches en phosphore, en fer, en magnésium, en manganèse, en zinc et en vitamine $B$ (Anilakumar et al., 2010). Le Tableau 4 illustre la composition en quelques paramètres biochimique de la graine de sésame dans six pays et selon des auteurs différents. Il est observé une différence entre les teneurs qui peut être due aux méthodes utilisées, aux facteurs environnementaux ou aux variétés.

\section{COMPOSITION ET PROFIL DES ACIDES GRAS}

En général, les profils d'acides gras des différentes variétés sont les mêmes dans la littérature pour le sésame. Cela indique l'existence d'un étroit contrôle génétique de la composition d'huile de base des graines. L'acide oléique est le composant principal des acides gras représentant 43,51 à $49,05 \%$ des acides gras totaux, suivi de l'acide linoléique $(36,10$ à $39,80 \%)$, l'acide palmitique $(7,83$ à $9,46 \%)$, l'acide stéarique $(5,40$ à $6,09 \%)$ et l'acide arachidonique $(0,31$ à $0,57 \%)$ (Ozkan et al., 2012). Ces fourchettes de composition en acides gras de la graine varient considérablement selon les génotypes et sous différentes conditions environnementales (Söğüt, 2008). Au total ces quatre acides représentent environ $98 \%$ des acides gras totaux des accessions de sésame. Les accessions dans leur ensemble affichent une variation significative pour les acides gras pris individuellement. Des gammes plus larges de variations ont été observées pour les acides oléique et linoléique, en contraste avec les autres acides gras. Une corrélation a été notée entre la teneur en huile et les valeurs trouvées pour les acides gras dans l'huile. Were et al. (2006) ont montré que la teneur en huile a été associée positivement avec les acides stéarique et oléique, mais avait une relation inverse avec les acides palmitique et linoléique. Cette forte tendance de variation opposée de ces acides gras est révélée par plusieurs auteurs dans d'autres spéculations comme le tournesol avec Flagella et al. (2002), le coton avec Liu et al. (2002), l'arachide avec Andersen et Gorbet (2002) mais aussi le colza d'hiver avec Möllers et 
Schierholt (2002). Ce qui laisse penser que des études pour la caractérisation biochimique en vue d'une sélection de variétés pour une homologation et une vulgarisation de variétés à haute teneur en huile de sésame devrait prendre en compte cette réduction concomitante des acides palmitique et linoléique, et une augmentation de l'acide oléique. D'autres acides sont aussi présents comme le montre dans le Tableau 5 le profil des acides gras rapporté par Yogranjan et al. (2014) ; montrant aussi la composition en acides gras saturés et insaturés dans l'huile de sésame. Ces auteurs montrent une prédominance des acide linoléique (C18: 2) et oléique (C18:1) et un taux élevé d'acides gras saturés comme le montre aussi Elleuch et al. (2007). Les niveaux élevés d'acides gras polyinsaturés (AGPI) et insaturés (AGS) augmentent la qualité de l'huile pour la consommation humaine (Nupur et al., 2010). Cette teneur en acides gras insaturés $(85,0 \%)$ est aussi trouvée par Latif et Anwar (2011). Cette composition en acides gras essentiels de l'huile additionnée à la fraction antioxydante serait en corrélation avec l'essor que connait le sésame en termes de prix dans le marché international. Les travaux de Nabloussi et al. (2017) ont aussi montré cette importance des graines avec un bon niveau d'antioxydant naturel des génotypes marocains

\section{UTILISATION DU SESAME}

Etant l'une des plus anciennes cultures oléagineuses dans le monde, le sésame fait aujourd'hui l'objet de diverses utilisations. Il est utilisé en alimentation, dans la pharmacologie (Anilkumar et al., 2010) et dans l'industrie avec des produits comme les parfums, les produits cosmétiques pour les chevaux et la peau, les huiles capillaires et les savons. En alimentation, l'huile est utilisée dans la cuisson, les graines sous formes de petits pains, croustilles de cracker de gâteaux, de soupe, d'épices, dans la confiture et dans la production de pétrole. Elles entrent également dans la production de la pâte (tehineh) et dans des formulations alimentaires tel que Halaweh (tehineh sucré) (Abou-Gharbia et al., 2000).
Le sésame contient de nombreux composés phytochimiques comme les flavonoïdes, les acides phénoliques, les alcaloïdes, les tanins, les saponines, les stéroïdes, les terpénoïdes... mais aussi les tocophérols et les lignanes qui lui confèrent plusieurs propriétés pharmaceutiques illustrées dans le Tableau 6 ci-dessous. D'autres auteurs ont également montré que la sésamine protège contre les lésions hépatiques (Nakai et al., 2003), et en raison de l'élévation de la concentration de la vitamine $\mathrm{E}$, diminue la péroxydation lipidique (Ikeda et al., 2003) et inhibe la production de superoxydes vasculaires (Nakano, 2003). L'autre application pharmaceutique déclarée de l'huile de sésame est son utilisation comme un bain de bouche antibactérien (Vishwanath et al., 2012) comme laxatif et dans le traitement de la sécheresse de la muqueuse nasale (Morris, 2002).

\section{INTERETS AGRONOMIQUES DE LA CULTURE DU SESAME}

En plus de ses intérêts économique, pharmaceutique, neutraceutique, et alimentaire, la culture du sésame comporte des avantages agronomiques dans le système de recadrage et de rotation des cultures. Anon (2002) a rapporté que la culture du sésame aurait plusieurs effets sur le sol. Elle réduit la population de nématodes qui attaquent le coton et l'arachide. Elle permet une excellente rotation des cultures de maïs, d'arachide, de blé et de sorgho. Elle est un excellent constructeur du sol, car améliorant la texture et la rétention de l'humidité du sol. Elle réduit l'érosion des sols par ses racines, qui sont en réseaux. En plus, les feuilles de sésame laissées sur le sol et compostées après culture, se lient au sol. La culture du sésame permet de mieux conserver l'humidité du sol par conséquent, augmente le rendement de la culture suivante.

Le sésame est une culture résistante à la sécheresse, tolérante aux insectes, aux ravageurs ainsi qu'aux maladies. 
Tableau 1: Documents consultés ou rencontres assistées lors de cette documentation.

\begin{tabular}{ll}
\hline Documents & Nombre \\
\hline Livres & 03 \\
Articles scientifique & 96 \\
Thèses & 11 \\
Mémoires & 15 \\
Rapport & 5 \\
Autres (séminaire, atelier, entretien ....) & 2 \\
\hline
\end{tabular}

Tableau 2: Répartition de la production nationale par région entre 2002 et 2006 au Sénégal (DAPS, 2007).

\begin{tabular}{lc}
\hline Régions & Taux de la production totale (\%) \\
\hline Diourbel & $5 \%$ \\
Kaolack & $42 \%$ \\
Kolda & $35 \%$ \\
Tambacounda & $7 \%$ \\
Ziguinchor & $5 \%$ \\
Autres régions & $6 \%$ \\
\hline Total & $100 \%$ \\
\hline
\end{tabular}

Tableau 3 : Caractéristiques de quelques variétés de sésame cultivées au Sénégal (Boureima, 2005).

\begin{tabular}{|c|c|c|c|c|c|c|}
\hline \multirow{2}{*}{ Variété } & \multirow{2}{*}{$\begin{array}{l}\text { Provena } \\
\text { nce }\end{array}$} & \multirow{2}{*}{$\begin{array}{l}\text { Cycle } \\
\text { (jours) }\end{array}$} & \multirow{2}{*}{$\begin{array}{l}\text { Type } \\
\text { botanique }\end{array}$} & \multicolumn{2}{|c|}{ Rendement $\left(\mathrm{kg} \mathrm{ha}^{-1}\right)$} & \multirow{2}{*}{ Couleur de la graine } \\
\hline & & & & Champ & Station & \\
\hline Ceraas-1-98 & Japon & 75 & Non ramifié & - & 625 & Blanche \\
\hline $32-15$ & Burkina & 90 & $\begin{array}{l}\text { Moyennement } \\
\text { ramifié }\end{array}$ & 686 & 1590 & Blanche \\
\hline $38-1-7$ & Burkina & 90 & $\begin{array}{l}\text { Moyennement } \\
\text { ramifié }\end{array}$ & - & 1455 & Crème \\
\hline Jaalgon 128 & Burkina & 90 & $\begin{array}{l}\text { Moyennement } \\
\text { ramifié }\end{array}$ & 559 & 1363 & Blanche \\
\hline Primoca & Sénégal & $110-120$ & Très ramifié & 843 & 879 & Brune \\
\hline
\end{tabular}


Tableau 4: Composition biochimique de la graine de sésame selon les auteurs et les régions.

\begin{tabular}{lllllll}
\hline \multicolumn{7}{c}{ Valeurs reportées dans la littérature } \\
\cline { 2 - 7 } & Maroc $^{\text {a }}$ & Soudan $^{\mathbf{b}}$ & Congo $^{\mathbf{c}}$ & Nigeria $^{\mathbf{d}}$ & Turquie $^{\mathbf{e}}$ & Egypte $^{\mathbf{f}}$ \\
\hline Matières grasses \% & $52 \pm 2.5$ & $47,4 \pm 0,4$ & $54 \pm 0,16$ & $36 \pm 5$ & $54,3 \pm 1$ & $56-60$ \\
humidité \% & $6 \pm 2.5$ & $3,75 \pm 0,1$ & $5,7 \pm 0,24$ & $6,5 \pm 0,7$ & $44 \pm 005$ & $02-3,06$ \\
Protéines \% & $22 \pm 1,5$ & $34,4 \pm 0,8$ & $20 \pm 0,12$ & $19 \pm 1$ & $21 \pm 0,1$ & $18,92-23$ \\
fibres totaux \% & $3,5 \pm 1,5$ & $3,76 \pm 0,1$ & $3,2 \pm 0,12$ & $17,9 \pm 0,3$ & - & $6,75-7,34$ \\
cendres totales \% & $4,5 \pm 0,5$ & $9 \pm 0,2$ & $3,7 \pm 0,92$ & $1,56 \pm 05$ & $4,41 \pm 005$ & $3,01-4,38$ \\
\hline
\end{tabular}

Références: a Gharby et al. (2015) ; b Sabah El Khier et al. (2008); c Nzikou et al. (2009); d Ogbonna and Ukaan (2013); e Unal and Yalc (2008); f Hassan (2012).

Tableau 5: Composition et profil des acides gras dans l'huile de sésame (Yogranjan et al., 2014).

\begin{tabular}{ll}
\hline Acides gras & Pourcentage dans l'huile (\%) \\
\hline Acide palmitique $(16: 0)$ & 11,7 \\
Acide stéarique $(18: 0$ & 05,2 \\
Acide oléique $(18: 1)$ & 41,4 \\
Acide linoléique $(18: 2)$ & 39,4 \\
Acide linolenique $(20: 3)$ & 00,4 \\
Acide arachidique $(20: 0)$ & 00,4 \\
Acide behenique $(22: 0)$ & 00,6 \\
Acides Gras saturés & 14,0 \\
Acide gras monoinsaturés & 39,0 \\
Acides gras polyinsaturés & 46,0 \\
Total acides gras insaturés & 85,0 \\
\hline
\end{tabular}

Tableau 6: Les propriétés pharmaceutique et nutraceutique des phytoconstituents du sésame.

\begin{tabular}{lll}
\hline $\begin{array}{l}\text { Composés } \\
\text { phytochimiques }\end{array}$ & Utilisations et Fonctions & Référence \\
\hline Lignanes : & Anti-mutagène, antioxydant, antihypertenseur, & Shad et al. (2007), ASGA \\
Sésamine, sésamol & $\begin{array}{l}\text { hypocholestérolémiant, anti-inflammatoire, anti- } \\
\text { sésaminol }\end{array}$ et & thrombotique, neuroprotecteur, cardioprotecteur, \\
composés & utilisé comme bactéricide et insecticide, et dans le et al. (2004); Miyake \\
apparentés & cas de l'hépatique accrue (mitochondriale et & (2009), Gauthaman \\
& l'oxydation des peroxysomes), & Bournival et al. (2012).
\end{tabular}




\begin{tabular}{|c|c|c|}
\hline Huile de sésame & $\begin{array}{l}\text { Adoucissant de la peau, améliore la résistance des } \\
\text { cellules à la peroxydation lipidique, véhicule } \\
\text { oléagineux pour les médicaments et les laxatifs, } \\
\text { utilisée dans le traitement de la sécheresse de la } \\
\text { muqueuse nasale, d'une vision floue, des } \\
\text { étourdissements, de l'anxiété, des maux de tête et de } \\
\text { l'insomnie, }\end{array}$ & $\begin{array}{l}\text { Kaur and Saini (2000); } \\
\text { Shivhare et Satsangee } \\
\text { (2012). }\end{array}$ \\
\hline Acide myristique & $\begin{array}{l}\text { Utilisé dans la prévention du cancer, et dans } \\
\text { l'industrie (cosmétiques et du savon) }\end{array}$ & $\begin{array}{l}\text { Morris (2002); Ahmad et } \\
\text { al. (2006) }\end{array}$ \\
\hline Flavonoïdes & Hypoglycémique & $\begin{array}{l}\text { Asgari et al. (2012); Morris } \\
\text { (2002) }\end{array}$ \\
\hline $\begin{array}{l}\text { Lécithine } \\
\text { lignanes }\end{array}$ & $\begin{array}{l}\text { Utilisés dans la diminution de la dermatite, } \\
\text { l'oxydation (rancissement) et l'inhibition de la } \\
\text { production de cholestérol }\end{array}$ & $\begin{array}{l}\text { Anilakumar et al. (2010); } \\
\text { Shivhare et Satsangee } \\
\text { (2012). }\end{array}$ \\
\hline Fibres & Utilisées comme cardio-protecteurs & $\begin{array}{l}\text { Jaffe (2012); Anilakumar } \\
\text { et al. (2010); Morris } \\
(2002) \text {. }\end{array}$ \\
\hline Chlorosesamone & Antifongique & $\begin{array}{l}\text { Chakraborthy et al. (2008); } \\
\text { Gauthaman (2009) }\end{array}$ \\
\hline Céphaline & Activité homéostatique & Anilakumar et al. (2010) \\
\hline
\end{tabular}

\section{Conclusion}

Le sésame est d'une grande importance dans le monde à cause des usages multiples de ses produits (graines, huile et tourteaux) : alimentation humaine et animal, propriétés thérapeutiques et industrielles. Cependant, au Sénégal, il est confronté à des contraintes qui limitent l'intensification de sa culture et sa valorisation. La présente synthèse qui a fait le point sur l'état de la recherche, sur les connaissances actuelles du sésame, notamment ses nombreuses vertus et propriétés de par sa composition en protéines, en matières grasses (acides gras saturés et insaturés) en antioxydants (sesamine et sesamoline sésamole) et en éléments minéraux montre la nécessité d'entreprendre au Sénégal des travaux futurs de recherche sur cette spéculation au Sénégal. Ainsi nos recherches seront orientées vers le contenu nutritionnel des génotypes de sésame au Sénégal mais aussi à l'étude de quelques composés phytochimiques comme les antioxydants et lignanes qui lui offrent de propriétés importantes pour la santé humaine. Il serait aussi importé d'étudier la biodisponibilité des éléments minéraux et le comportement de l'huile obtenue par presse des variétés de sésame Sénégalaises face à la température de cuisson de même qu'au contenu antinutritionnel (phytates, oxalates et les alpha-galactosides. Le seul but sera de développer la culture de sésame afin d'accroitre sa consommation et les revenus des populations rurales du fait de l'essor qu'il connait actuellement sur le marché mondial. 


\section{CONFLITS D'INTERETS}

Les auteurs déclarent n'avoir aucun intérêt concurrent.

\section{REFERENCES}

Abou-Gharbia H A, Shehata AAY, Shahidi F. 2000. Effect of processing on oxidative stability and lipid classes of sesame oil. Food Research International, 33: 331340. DOI: agris.fao.org/agrissearch/search.do?recordID=US20160009 7266.

Ahmad S, Yousuf S, Ishrat T, Khan M B, Bhatia K, Fazli I S and Islam F (2006). Effect of dietary sesame oil as antioxidant on brain hippocampus of rat in focal cerebral ischemia, Life sciences, 79 (20): 1921-1928.

Andersen PC, Gorbet DW. 2002. Influence of year and planting date on fatty acid chemistry of high oleic acid and normal peanut genotypes. J. Agric. Food Chem. 50:

1298-1305.

DOI: $10.1021 /$ jf0113171

Anilakumar K, Pal A, Khanum F, Bawa A. 2010. Nutritional, Medicinal and Industrial Uses of Sesame (Sesamum indicum L.) Seeds - An Overview. Agriculturae Conspectus Scientificus. 75(4): 159-168. DOI: https://hrcak.srce.hr/66001.

Anonymous. 2002. Overview of the Nigerian Sesame industry. Prepared for the United States Agency for International Development (USAID)/Nigeria. Chemonic International Inc., Washington DC, USA. 34 p.

ASGA Sesame Markets [online]. 2011. Available from: http://www.sesame growers.org/usesofsesame.htm.

[Accessed 27/08/2017].

Asgari S, Setorki M, Rafieian-kopaei M, Heidarian E, Shahinfard N, Ansari R, Forouzandeh Z. 2012. Postprandial hypolipidemic and hypoglycemic effects of Allium hertifolium and Sesamum indicum on hypercholesterolemic rabbits.
Afr J Pharm Pharmacol., 6 (15): 11311135.

DOI: https://doi.org/10.5897/AJPP12.09 2.

Ashri A. 2001. Induced mutations in sesame breeding. In: Sesame improvement by induced mutations, IAEA-TECDOC1195, IAEA, Vienna, 13-20.

Ashri A. 2007. Sésame (Sesamum indicum L.). Cultures oléagineuses. In Les Ressources Génétiques, l'Ingénierie des Chromosomes et l'Amélioration des Cultures (Volume 4), Singh RJ (Ed). CRC Press: Boca Raton, Floride, États-Unis ; 231-289.

Bèye G. 2003. Effet de la densité de semis sur la croissance et la productivité de deux types botaniques du sésame (Sesamum indicum L.) au Sénégal. Mémoire d'ingénieur agronome, Ecole Nationale Supérieure d'Agriculture (ENSA), Thiès, $59 \mathrm{p}$.

Biswa TK, Sana NK, Badal RK, Huque Entazul M. 2001. Biochimical study of some oil seeds (Brassica, Sesame and linseed). Pakistan Journal of Biological Sciences, $\quad \mathbf{4}(8)$ : $\quad$ 1002-1005. DOI: $10.3923 /$ pjbs.2001.1002.1005

Borchani C, Besbes S, Blecker CH, Attia H. 2010. Chemical Characteristics and Oxidative Stability of Sesame Seed, Sesame Paste, and Olive Oils. Journal of Agriculture, Science and. Technology, 12: 585-596. DOI : http://journals.modares.ac.ir/articl e-23-7694-fr.html

Boureima S. 2005. Besoins en eau, croissance et productivité chez le type botanique ramifié du sésame (Sesamum indicum L.) en zone semi-aride du sénégal. Mémoire d'ingénieur agronome, Ecole Nationale Supérieure d'Agriculture (ENSA), Thiès, 60p.

Boureima S. 2012. Amélioration variétale du sésame (Sesamum indicum L.) par mutation induite : effet de la mutagenèse sur la tolérance à la sécheresse et la 
productivité. Thèse de doctorat. Faculty of Bioscience Engineering, Ghent University, Belgium 194 pp.

Bournival J, Francoeur MA, Renaud J, Martinoli MG. 2012. Quercetin and sesamin protect neuronal PC12 cells from high-glucose-induced oxidation, nitrosative stress, and apoptosis. Rejuvenation Research, 15 (3): 322-333. DOI: $10.1089 /$ rej.2011.1242.

Brito OJ, Núñez N. 1982. Evaluation of sesame flour as a complementary protein source for combinations with soy and corn flours. Journal of Food Science, 47(2): 457-460. https://doi.org/10.1111/j.13652621.1982.tb10103.x.

Burden D. 2005. Sesame Profile. Available at http//: www.cropprofile.mht.accessed 15 January, 2008.

Chakraborthy G S, Sharma G and Kaushik K N. 2008. Sesamum indicum: a review. Journal of Herbal Medicine and Toxicology, 2 (2): 15-19.

CRS (Catholic Relief Services). 1999. Actes $\mathrm{du}$ Forum des acteurs de la filière sésame, du 15 au 18 juin à Faoune, Région de Kolda, 53p

DAPS (Direction de l'Analyse, de la Prévision et des Statistiques). 2006. Rapport de l'USAID/CE sur la chaine de valeur sésame au Sénégal: analyse et cadre stratégique d'initiatives pour la croissance de la filière, 69pp.

Deme T, Haki GD, Retta N, Woldegiorgis A, Geleta M. 2017. Mineral and AntiNutritional Contents of Niger Seed (Guizotia abyssinica (L.f.) Cass., Linseed (Linum usitatissimum L.) and Sesame (Sesamum indicum L.) Varieties Grown in Ethiopia. Foods, 6(27) ; DOI:10.3390/foods6040027.

Demol J, Baudoin JP, Louant BP, Maréchal R, Mergeai G, Otoul E. 2002. Amélioration des Plantes: Application aux Principales Espèces Cultivées en Régions
Tropicales. Les Presses Agronomiques de Gembloux : Gembloux ; 581 p.

Diouf M. 2002. Effet de l'interaction génotype $\mathrm{x}$ milieu sur la croissance et la productivité du sésame (Sesamum indicum L.) au Sénégal. Mémoire d'ingénieur agronome, Ecole Nationale Supérieure d'Agriculture (ENSA), Thiès, 93 p. + ann.

Diouf M, Fofana A, Ndiaye M, Guèye M. 2004. Fiche technique du sésame, ISRACERAAS, Thiès, $3 p$.

Diouf M, Sarr B, Diop M. 2002. Esquisse de carte variétale du sésame au Sénégal. Isra-Ceraas, Thiès, $1 \mathrm{p}$.

Diouf M. 2004. Programme de formation et de recherche-développement pour la relance de la filière sésame (Sesamum indicum L.) au Sénégal, ISRA-CERAAS, Thiès, $21 \mathrm{p} .+$ ann.

Elleuch M, Besbes S, Roiseux O, Blecke C H, Attia. 2007. Quality characteristics of sesame seeds and by-products. Food Chemistry, 103: 641-650. DOI: 10.1016/j.foodchem.2006.09.008

FAO (Organisation des Nations Unies pour l'alimentation et l'agriculture). 2004. Statistiques principales du commerce extérieur agro-alimentaire. FAO. www.fao.org/es/ess/toptrade/trade.asp

FAOSTAT, 2011. FAO Statistics Division 2011; Sesame seed. In Food and Agriculture Organization of the United Nation. FAOSTAT.

Flagella Z, Rotunno T, Tarantino E, Di Caterina A, De Caro A. 2002. Changes in seed yield and oil fatty acid composition of high oleic sunflower (Helianthus annuus L.) hybrids in relation to the sowing date and water regime. Eur. J. Agron., 17: 221-230. DOI : $\quad$ https://doi.org/10.1016/S11610301(02)00012-6.

Fofana A. 2004. Evaluation variétale chez le sésame, Rapport annuel de projet, ISRACRZ, Kolda, 13p. 
Gandhi AP. and Srivastava J. 2007. Studies on the production of protein isolates from defatted sesame seed (Sesamum indicum) flour and their nutritional profile. ASEAN Food Journal, 14(3): 175-180. www.ifrj.upm.edu.my/afjv14(3)2007/17 5-180.pdf

Gauthaman K. 2009. Nutraceutical value of sesame oil. Pharmacognosy Reviews, 3(6): 264.2 DOI: http://www.phcogrev.com/text.asp?2009 /3/6/264/59526

Gharby S, Harhar H, Bouzouba Z, Asdadi A, El Yadini A, Charrouf Z. 2015. Chemical characterization and oxidative stability of seeds and oil of sesame grown in Morocco. Journal of the Saudi Society of Agricultural Sciences, 16: 105-111.

DOI: https://doi.org/10.1016/j.jssas.2015.03.0 04.

Guèye M. 2002. Réponse morphophysiologique au déficit hydrique du sésame (Sesamum indicum) au jeune âge. Mémoire de DEA, Université Cheikh Anta Diop de Dakar (UCAD), 45 p.

Hassan Manal AM. 2012. Studies on Egyptian sesame seeds (Sesamum indicum L.) and its products 1 - physicochemical analysis and phenolic acids of roasted Egyptian sesame seeds (Sesamum indicum L.). World J. Dairy Food Sci., 7 (2): 195-201. DOI: 10.5829/idosi.wjdfs.2012.7.2.1112.

Ikeda S, Kagaya M, Kobayashi K, Tohyama $\mathrm{T}$, Kiso Y, Higuchi N, Yamashita K. 2003. Dietary sesame lignans decrease lipid peroxidation in rats fed docosahexaenoic acid. J. Nutr. Sci. Vitaminol, 49(4): 270-276. DOI: https://doi.org/10.3177/jnsv.49.270

Iwe MO, van Zuilichem DJ, Ngoddy PO, Lammers W. 2001. Amino acid and protein dispersibility index (PDI) of mixtures of extruded soy and sweet potato flours. $L W T$ - Food Science and
Technology, $\quad \mathbf{3 4}(2): \quad 71-75 . \quad$ DOI: https://doi.org/10.1006/fstl.2000.0731

Jaffe R. 2012. Cardioprotective Nutrients, Bioactive Food as Dietary Interventions for Cardiovascular Disease: Bioactive Foods in Chronic Disease States, pp 103. Kafiriti EM, Deckers J. 2001. Sésame : Sesamum indicum L. In Raemaekers, Agriculture en Afrique Tropicale. Direction Générale de la Coopération Internationale (DGCI): Bruxelles, Belgique ; $1634 \mathrm{p}$.

Kaur IP, Saini A. 2000. Sesamol exhibits antimutagenic activity against oxygen species mediated mutagenicity. Mutation Research, 470: 71-76. DOI:10.1016 / S1383-5718 (00) 00096-6.

Latif S, Anwar F. 2011. Aqueous enzymatic sesame oil and protein extraction. Food Chem., 125: 679-684. DOI:10.1016/j.foodchem.2010.09.064.

Lee Edwald, Eunok Choe. 2012. Changes in oxidation-derived off-flavor compounds of roasted sesame oil during accelerated storage in the dark. Biocatalysis and Agricultural Biotechnology, 1: 89-93. DOI: $10.1016 /$ j.bcab.2011.08.003.

Liu Q, Singh PS, Green AG. 2002. Highstearic and high-oleic cottonseed oils produced by hairpin RNA-Mediated Post-Transcriptional Gene silencing. Plant Physiol., 129: 1732-1743. DOI: https://doi.org/10.1104/pp.001933.

Mémento de l'Agronome, 2002. Mémento de l'Agronome. Ministère des affaires étrangères, Centre de coopération internationale en recherche agronomique pour le développement (Cirad), Groupe de recherche et d'échange technologique (Gret), Paris, $1690 \mathrm{p}$.

Miyake Y, Fukumoto S, Okada M, Sakaida K, Nakamura Y and Osawa T. 2005. Antioxidative catechollignans converted from sesamin and sesaminol triglucoside by culturing with Aspergillus. Journal of Agricultural and Food Chemistry, 53 (1): 22-27: DOI: 10.1021 / jf048743h. 
MöllersMo“llers C, Schierholt A. 2002. Genetic variation of palmitate and oil content in a winter oilseed rape doubled haploid population segregating for oleate content. Crop Sci., 42: 379-384. DOI:10.2135/cropsci2002.3790.

Morris JB. 2002. Food, industrial, nutraceutical, and pharmaceutical uses of sesame genetic resources, In Trends in New Cropsand New Uses, Alexandria VA (Eds.). ASHS Press; 153-156.

Nabloussi A, Hanine H , Harfi ME, Rizki1H. 2017. Moroccan sesame: an overview of seed and oil quality, Science within Food: Up-to-date Advances on Research and Educational Ideas (1) Food Science Book.

Nakai M, Harada M, Nakahara K, Akimoto K, Shibata H, Miki W, Kiso Y . 2003. Novel antioxidative metabolites in rat liver with ingested sesamin, J. Agric. Food Chem., 51(6): 1666-1670. DOI: https://pubs.acs.org/doi/abs/10.1021/jf02 58961.

Nakano D, Itoh C, Ishii F, Kawanishi H, Takaoka M, Kiso Y, Tsuruoka N, Tanaka T, Matsumura Y. 2003. Effects of sesamin on aortic oxidative stress and endothelial dysfunction in deoxycorticosterone acetate-salt hypertensive rats, Biol. Pharm. Bull, 26 (12): 1701-1705. DOI : https://www.ncbi.nlm.nih.gov/pubmed/1 4646174.

Ndiaye M, Mbaye K, Fofana A, Fall A, Diouf M. 2005. Guide de production du sésame (Sesamum indicum L.) en pluvial au Sénégal. Editions ISRA, 25 pages.

Ndiaye M. 2004. Effets de la fertilisation minérale sur la croissance, le développement et la productivité du sésame (Sesamum indicum L.) cultivé au Sénégal. Rapport annuel de projet, IsraCNRA, Bambey, 9p.

Niang M. 2004. Effet de la date de semis sur le développement, la croissance et la productivité du sésame (Sesamum indicum L.) en zone semi-aride du Sénégal. Mémoire d'ingénieur agronome, Ecole Nationale Supérieure d'Agriculture (ENSA), Thiès, 60p. + Annexe.

Nupur M, Bhat MV, Srivastava PS. 2010. Variation in fatty acid composition in Indian germplasm of sesame. Journal of the American Oil Chemists' Society, 87(11): $\quad$ 1263-1269. DOI: https://doi.org/10.1007/s11746-0101615-9.

Nzikou JM, Matos L, Bouanga-Kalou G, Ndangui CB, Pambou-Tobi NPG, Kimbonguila A, Silou T, Linder M, Desobry S. 2009. Chemical Composition of the Seeds and Oil of Sesame (Sesamum indicum L.) Grown in CongoBrazzaville. Advance Journal of Food Science and Technology, 1(1): 6-11. http://maxwellsci.com/print/ajfst/611.pdf.

Nzikou JM, Mvoula-Tsiéri M, Ndangui CB, Pambou-Tobi NPG, Kimbonguila A, Loumouamou B, Silou T, Desobry S. 2010. Characterization of Seeds and Oil of Sesame (Sesamum indicum L.) and the Kinetics of Degradation of the Oil During Heating. Research Journal of Applied Sciences, Engineering and Technology, 2(3): 227-232.

Nabloussi A, Hanine H, Harfi ME, Rizki H. 2018. Moroccan sesame: an overview of seed and oil quality. In Science within Food: Up-to-date Advances on Research and Educational Ideas (1). Food Science Book.

Ogbonna PE, Ukaan SI. 2013. Chemical composition and oil quality of seeds of sesame accessions grown in the Nsukka plains of South Eastern Nigeria. Afr. J. Agric. Res., 8(9): 797-803. DOI: https://doi: 10.5897/AJAR12.1702

Onsaard E, 2012. Sesame proteins. International Food Research Journal 19(4): 1287-1295. http://www.ifrj.upm.edu.my 
Ozkan A, Deniz C, Muhittin K. 2012. Morphological properties and chemical compositions of some sesame (Sesamum indicum L.) populations cultivated in Kilis, Turkey. African Journal of Agricultural Research, 7(19): 30293033.

DOI : https://doi.org/10.5897/AJAR11.2245.

Pelletier S. 2003. Effet du stress hydrique sur le comportement agro-morphologique et physiologique du sésame (Sesamum indicum L.) de type variété cultivé en champ. Rapport de stage de pré-option, Ecole Nationale des Ingénieurs des Travaux Agricoles/Centre d'étude régional pour l'amélioration de l'adaptation à la sécheresse, Bordeaux, $47 \mathrm{p}$.

Peter RS. 2007. Improving the protein content and composition of cereal grain. Journal of Cereal Science, 46(3): 239-250. DOI: 10.1016/j.jcs.2007.06.006.

Raghav PK, Agarwal N. 2013. Comparison of sensory qualities of three popular brands of gajaks sold in jaipur market. International Journal of Agricultural and Food Science, 4(1): 6-8, https://www.urpjournals.com/tocjnls/7_1 4v4i1_2.pdf.

Rizki H, Kzaiber F, Elharfi M, Nablousi A, Ennahli S, Hanine H. 2015. ! Assessment of antioxidant capacity of 16 cultivars of sesame (Sesamum indicum.L) from different areas, International Journal of Innovation and Scientific Research, 18(2): 379-385. DOI:

http://citeseerx.ist.psu.edu/viewdoc/dow nload?doi=10.1.1.830.9066\&rep=rep1\&t ype $=$ pdf.

Rizki H, Nabloussi A, Kzaiber F, Latrache H, Hanine H. 2017. Mineral Composition of Some Accessions of Sesame Seeds (Sesamum Indicum L.) Collected from Morocco. International Journal of Engineering Research and Allied
Sciences, 2(8): 2455-9660. DOI: $10.17957 / \mathrm{IJAB} / 15.0145$.

Sabah El Khier MK, Khogali Elnur AI, Abu El Gasim AY. 2008. Chemical composition and oil characteristics of sesame seed cultivars grown in Sudan. Res. J. Agric. Biol. Sci., 4(6): 761-766. www.aensiweb.net/AENSIWEB/rjabs/rj abs/2008/761-766.pdf.

Saha R, Dinar AM, Nabila K A, Roy P. 2014. HPLC analysis and cell surface receptor binding activities of the crude aqueous and methanolic extract of Sesamum indicum. Asian Pac. J. Trop. Biomed., 4(1): S516-S520.

DOI: 10.12980/APJTB.4.2014C973.

Sene B, Sarr F, Sow M S, Diouf D, Niang M, Traoré D. 2017. Physico-Chemical Composition of the Sesame Variety (Sesamum indicum L.) $32-15$ and Characterization of its Derived Products (Seeds, Oil and Oilcake) in Senegal. Food Science and Quality Management, 65(2017): 2225-0557. DOI: http://www.iiste.org/Journals/index.php/ FSQM/article/view/37829.

Shad KF, Al-Salam S, Hamza AA. 2007. Sesame oil as a protective agent against doxorubicin induced cardio toxicity in rat. American Journal of Pharmacology and Toxicology, 2(4): 159-163. DOI:10.3844/ajptsp.2007.159.163.

Shivhare N, Satsangee N. 2012. Wonders of Sesame: Nutraceutical Uses and Health Benefits. In Chemistry of Phytopotentials: Health, Energy and Environmental Perspectives. Springer: Berlin Heidelberg; 63-68.

Söğüt T. 2008. Effect of main and second cropping on seed y1eld, oil and protein content of Sesame (Sesamum indicum L.) Genotypes. Tukish J. Field Crops, 14(2): 64-71. DOI: http://dergipark.gov.tr/download/articlefile/158790.

Stevens PF. 2011. Angiosperm Phylogeny Website. 
http://www.mobot.org/mobot/research/a pweb/ consulté le 14 Février 2017.

Suja KP, Jayalekshmy A, Arumughan C. 2004. Free radical scavenging behavior of antioxidant compounds of sesame (Sesamum indicum L.) in DPPH system. Journal of Agricultural and Food Chemistry, $\quad$ 52(4): 912-915. DOI: 10.1021 / jf0303621.

Unal MK, Yalc INH. 2008. Proximate composition of Turkish seeds and characterization of their oils. Grasas $Y$ Aceites 59(1): 23-26. DOI : http://dx.doi.org/10.3989/gya.2008.v59.i 1.485.

USAID-CE. 2008. Analyse et cadre stratégique d'initiatives pour la croissance de la filière sésame, janvier 69p-Annexe.

Valarmathi G, Surendran C, Vanniarajan C, Kumar M, Saravanan NA. 2003. Morphological and biochemical characterization of sesame (sesamum indicum 1. and s. mulayanum 1.). Sesame and Safflower, Newsletter, 18: 42-46. https://www.cabdirect.org/cabdirect/abst $\mathrm{ract} / 20093312505$.

Vishwanath H, Anilakumar KR, Harsha SN, Khanum $\mathrm{F}$ and Bawa AS. 2012. In vitro antioxidant activity of Sesamum indicum seeds. Asian Journal of Pharmaceutical and Clinical Research, 5(1): 56-60. https://innovareacademics.in/journal/ajpc r/Vol5Suppl1/658.pdf.

Weiss EA. 2000. Oilseed Crops (second edition). Blackwell Science LTD: United Kingdom; 355p.

Were BA, Onkware AO, Gudu S, Welander M, Carlsson AS. 2006. Seed oil content and fatty acid composition in East African sesame (Sesamum indicum L.) accessions evaluated over 3 years. Field Crops Research, 97: 254-260. DOI:10.1016/j.fcr.2005.10.009.

Yogranjan, Satpute GK, Marabi RS, Manish KM, Mishra SP. 2014. Global resurgence of sesame (Sesamum indicum L.) utilization: a current scenario. IndoAm. J. Agric. \& Vet. Sci, 2(3) : 23219602.

http://www.iajavs.com/currentissue.php 